\title{
miR-205-5p-mediated downregulation of ErbB/HER receptors in breast cancer stem cells results in targeted therapy resistance
}

\author{
A De Cola ${ }^{1}$, S Volpe ${ }^{2}$, MC Budani ${ }^{1}$, M Ferracin ${ }^{3}$, R Lattanzio ${ }^{1}$, A Turdo ${ }^{2}$, D D’Agostino ${ }^{1}$, E Capone ${ }^{1}$, G Stassi ${ }^{2}$, M Todaro ${ }^{2}$, C Di llio ${ }^{1}$, \\ G Sala ${ }^{1,4}$, M Piantelli $^{1}$, M Negrini $^{3}$, A Veronese $^{1}$ and V De Laurenzi ${ }^{\star, 1}$
}

The ErbB tyrosine kinase receptor family has been shown to have an important role in tumorigenesis, and the expression of its receptor members is frequently deregulated in many types of solid tumors. Various drugs targeting these receptors have been approved for cancer treatment. Particularly, in breast cancer, anti-Her2/EGFR molecules represent the standard therapy for Her2positive malignancies. However, in a number of cases, the tumor relapses or progresses thus suggesting that not all cancer cells have been targeted. One possibility is that a subset of cells capable of regenerating the tumor, such as cancer stem cells (CSCs), may not respond to these therapeutic agents. Accumulating evidences indicate that $m i R-205-5 p$ is significantly downregulated in breast tumors compared with normal breast tissue and acts as a tumor suppressor directly targeting oncogenes such as Zeb1 and ErbB3. In this study, we report that miR-205-5p is highly expressed in BCSCs and represses directly ERBB2 and indirectly EGFR leading to resistance to targeted therapy. Furthermore, we show that miR-205-5p directly regulates the expression of $p 63$ which is in turn involved in the EGFR expression suggesting a miR-205/p63/EGFR regulation.

Cell Death and Disease (2015) 6, e1823; doi:10.1038/cddis.2015.192; published online 16 July 2015

Breast cancer is the most frequent type of cancer in women and despite the great improvement in diagnosis and treatment, relevant number of patients eventually relapses (SEER Cancer Statistics Review, 1975-2007, National Cancer Institute. Bethesda, MD, http://seer.cancer.gov/csr/1975_2007/, based on November 2009 SEER data submission, posted to the SEER web site, 2010). Recent studies have provided strong support for the cancer stem cell (CSC) hypothesis which holds that breast cancers are driven by a subpopulation of cells within the tumor which display stem cell properties. ${ }^{1}$ These properties include self-renewal, which generates other CSCs and differentiation, which generates populations of cells forming the bulk of the tumor. There is increasing evidence that CSCs are relatively quiescent cells, resistant to chemotherapy and radiation therapy and can therefore contribute to treatment resistance and relapse. It is therefore possible that relapses observed in ErbB2-positive breast cancer patients receiving adjuvant Trastuzumab (humanized antibody antiHer2-Herceptin) or Lapatinib (small tyrosine kinases inhibitor molecule), ${ }^{2,3}$ is due to the presence of CSCs that escape these therapeutic agents. Various mechanisms have been reported to cause resistance to targeted therapy, such as reduced ErbB2 expression, increased pro-survival signaling through alternative tyrosine kinases receptors or altered intracellular signaling leading to cellular over-proliferation. ${ }^{4,5}$

Virtually all human genes are targeted by miRNAs, ${ }^{6}$ a class of non-coding endogenous small RNAs, which modulate the expression of their target genes through base pairing with the $3^{\prime}$ untranslated sequence ( $3^{\prime}$-UTR) of their target mRNAs. ${ }^{7,8}$ MiRNA deregulation is widely described in cancer and has an important role in tumorigenesis. 9,10

MiR-205-5p is a highly conserved miRNA, expressed in stratified squamous epithelial-derived tissues ${ }^{11}$ and in mammary gland progenitor. $^{12}$ It has been shown that miR-205-5p is downregulated in breast cancer and can specifically suppress ErbB3 expression. ${ }^{13}$ Moreover, miR-205-5p has been reported to mediate the epithelial to mesenchymal transition by targeting ZEB1 and ZEB2,$^{14,15}$ and it has a role in targeting several regulators of proliferation ${ }^{16,17}$ suggesting its involvement in cellular differentiation, migration and proliferation. In addition, it has been reported that miR-205-5p is regulated by p63, a p53 family member resulting in epithelial to mesenchymal transition inhibition, ${ }^{18}$ whereas the loss of the p63/miR-205 axis enhances cell migration and metastasis in prostate cancer cells. ${ }^{19}$

The TP63 gene contains two promoters that produce two proteins: the full-length TAp63 that contains functional $\mathrm{N}$-terminal transcriptional transactivation (TA) domains and the $\Delta$ Np63 protein, which lacks TA domains. ${ }^{20}$ p63 has central roles in epithelial development and despite the two isoforms share some common features, ${ }^{20}$ TAp63 mainly acts as tumor suppressor and $\Delta \mathrm{Np} 63$ as an oncogene. ${ }^{21,22}$

Here, we show that $m i R-205-5 p$ is upregulated in patient-derived breast CSCs (BCSCs), compared with more

\footnotetext{
${ }^{1}$ Department of Experimental and Clinical Sciences, Aging Research Center (Ce.S.I.), University G. D'Annunzio, Chieti-Pescara, Italy; ${ }^{2}$ Department of Surgical and Oncological Sciences, University of Palermo, Palermo, Italy; ${ }^{3}$ Department of Morphology, Surgery and Experimental Medicine and Laboratory for Technologies of Advanced Therapies (LTTA), University of Ferrara, Ferrara, Italy and ${ }^{4}$ Mediapharma s.r.l., Via dei Vestini, Chieti, Italy

*Corresponding author: V De Laurenzi, Department of Experimental and Clinical Sciences, Aging Research Center (Ce.S.I.), University G. d’Annunzio, Chieti-Pescara, Via Luigi Polacchi 11, Chieti, 66100, Italy. Tel: +39 0871 541580; Fax:+39 0871 541598; E-mail: delaurenzi@ unich.it

Abbreviations: BCSC, breast cancer stem cells; CSC, cancer stem cells; HT, hercept test; SDAC, sphere-derived adherent cells

Received 25.5.15; accepted 28.5.15; Edited by E Baehrecke
} 
differentiated tumor cells. More importantly, we show that miR-205-5p controls CSC phenotype targeting ErbB2, p63 and EGFR, contributing to targeted therapy resistance.

\section{Results}

BCSCs show low levels of ERBB2 and EGFR. We characterized three patient-derived BCSC lines (BCSC \#1, BCSC \#2, BCSC \#3) from three ErbB2-positive primary tumors. Immunohistochemistry analysis (Figure 1a) of the primary tumor confirms ErbB2 positivity in all three tumors, whereas CSCs derived from the tumors stain negative. Interestingly, when cells are grown as differentiating spherederived adherent cells (SDACs) for 14 days, they begin to show a faint positive staining. Western blot analysis confirms that spheroids retain very low expression levels of both ErbB2 and EGFR receptors that significantly increase when cells were grown as SDACs (Figure 1b). FACS analysis (Supplementary Figure S1) further confirms that indeed all three cell lines express variable (but generally low) levels of ErbB2 that again increase when cells are grown as SDACS. Interestingly, there is little correspondence between mRNA (Figure 1c) and protein levels for ErbB2 and almost none for EGFR, suggesting that expression changes in BCSCs and SDACs are at least in part mediated by non-transcriptional mechanisms. These data suggest that although CSCs show low expression levels of ErbB2 and EGFR, these receptors increase in tumor cells when they acquire a more differentiated phenotype.

BCSCs are resistant to Lapatinib. We then tested the sensitivity of cell lines grown as mammospheres to Lapatinib, an ATP-competitive reversible small-molecule inhibitor of the ErbB2 and EGFR tyrosine kinases currently used in clinics as therapy for Her2-overexpressing metastatic breast cancers resistant to Trastuzumab-based regimens. ${ }^{23}$ As shown in Figure 1d, cells are resistant to treatment consistently with low expression levels of the receptors.

We then investigated whether cells regain sensitivity to this treatment when they are grown as SDAC and increase receptors expression. Indeed although BCSCs are completely resistant to treatment with Lapatinib $(0.5 \mu \mathrm{M})$, the same cells grown as SDACs appear significantly more sensitive (Figure 1d). These results suggest that failure of therapies to target BCSCs could be at least in part due to reduced expression of the receptors.

There have been a number of studies describing a role of miRNA in the regulation of protein expression in normal and tumor breast stem cells. Moreover, a number of miRNAs have been shown to be involved during carcinogenesis, ${ }^{24,25}$ and emerging evidence suggests that miRNAs also have essential roles in stem cell self-renewal and differentiation by negatively regulating the expression of key genes. ${ }^{25}$ Therefore, we performed a miRNA expression profile to identify miRNAs differentially expressed in BCSCs (BCSC \#1) and SDACs collected at the indicated time point (7 days), and we identified 58 human miRNAs significantly differentially regulated (adjusted $P<0.05$ ). Among these, several have been previously shown to be involved in breast malignancy.
Supplementary Figure S2 shows miRNAs that presented the highest differences between BCSCs and SDACs. We focused our attention on miR-205-5p because it has been previously reported $^{12,26-29}$ to have a role in both normal mammary development and in breast cancer. MiR-205 has also been shown to be highly expressed in stem cell-enriched populations from normal mouse mammary gland, and thus may have a function also in maintaining the BCSC phenotype. We confirmed a higher expression of miR-205-5p in BCSCs as compared with SDACs in all three cell lines (Figure 2a). This inversely correlates with expression of ErbB2 and EGFR, thus suggesting that miR-205 is potentially a regulator of these receptors.

miR-205-5p regulates ERBB2 and EGFR expression. To investigate whether miR-205-5p is indeed capable of regulating ErbB2 and EGFR expression, we silenced it in BCSC by cloning the miR-205-5p mature sequence in a pSIH-H1 shRNA expression lentivector. As shown in Figure 2b, miR-205-5p knockdown results in a significant EGFR and ErbB2 upregulation at protein levels as well as at mRNA levels (Figure 2c). ZEB-1, a well-established miR-205-5p target, was used as a control to confirm functional miR-205-5p silencing.

In addition, overexpression of miR-205-5p in BCSC \#1 cells by infection with pCMV-RFP-2A-puro lentivector results in strongly reduced protein levels (Figure $2 \mathrm{~d}$ ) and in reduced mRNA levels (Figure 2e) of both ErbB2 and EGFR. Similar results were obtained using the same constructs in the other two BCSC lines (data not shown), suggesting a possible direct regulation of these two genes by $m i R-205-5 p$.

Because the main algorithms for miRNA target prediction fail to find the EGFR and ErbB2 as target of miR-205, we performed an in silico analysis using the RNAhybrid algoritm (http://bibiserv.techfak.uni-bielefeld.de/rnahybrid/submission. html) to identify putative target sites for miR-205-5p in the 3'-UTR of human ErbB2 (NM_004448) (Figure 2f) and of human EGFR (Figure 2g) (NM_005228). We found few putative binding region, therefore we cloned both ErbB2 and EGFR $3^{\prime}$-UTR containing the predicted miR-205-5p binding site into the pGL3 control vector downstream of the luciferase open reading frame. Co-transfection of mimic premiR-205 and the WT ErbB2 3'-UTR construct in SKBR3 cells results in a significant inhibition of the luciferase activity compared with cells in which the WT ErbB2 $3^{\prime}$-UTR construct was co-transfected with a control vector (Figure $2 \mathrm{f}$ ). Mutation of miR-205-5p binding site within the ErbB2 3'-UTR (ERBB2$3^{\prime}$-UTR Mut) abolishes the ability of $m i R-205-5 p$ to regulate the luciferase expression resulting in the increase of luciferase activity (Figure 2f). In contrast, different results were obtained co-transfecting miR-205-5p and the WT EGFR 3'-UTR construct in SKBR3 cells. Indeed, as shown in Figure $2 \mathrm{~g}$, miR-205-5p was not able to decrease luciferase activity, even at longer time points (24, 48 and $72 \mathrm{~h}$ after transfection). These data suggest that $m i R-205-5 p$ might modulate EGFR expression indirectly, by targeting others key factors involved in EGFR regulation.

miR-205-5p controls p63 expression. To further investigate the indirect regulation of EGFR expression by miR-205-5p, 
a
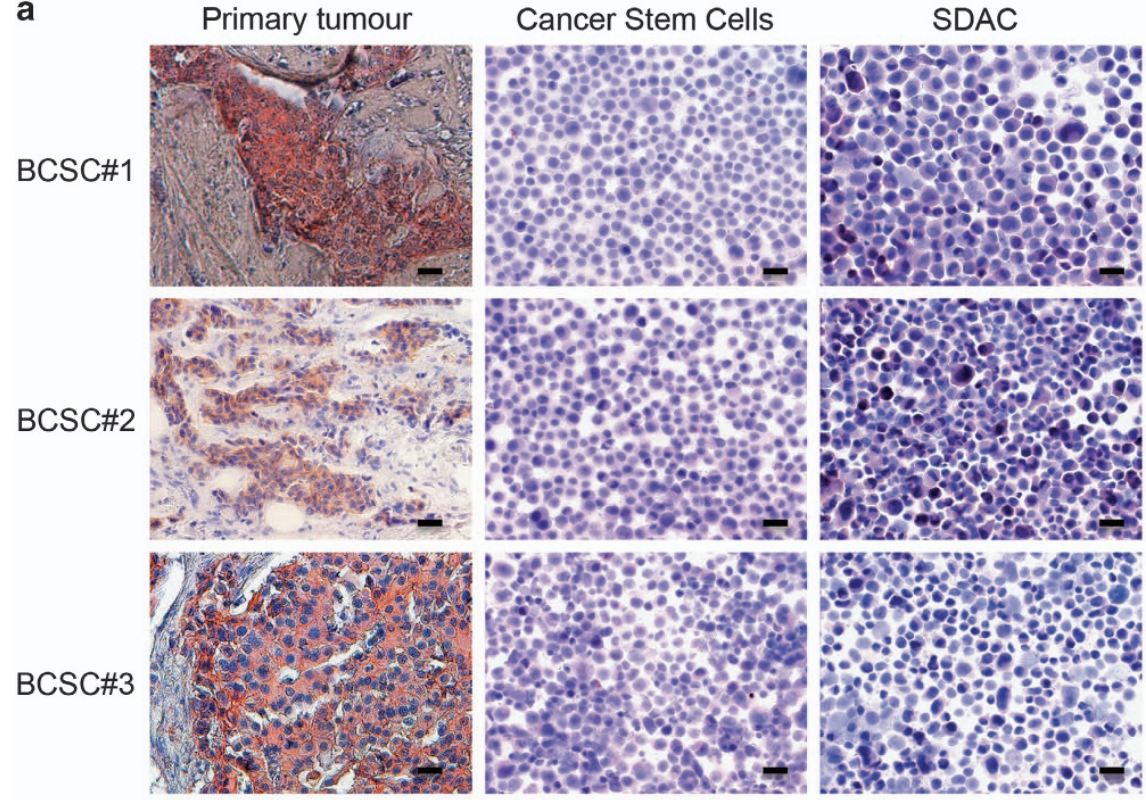

b

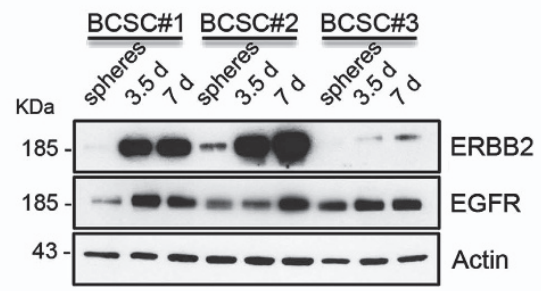

C
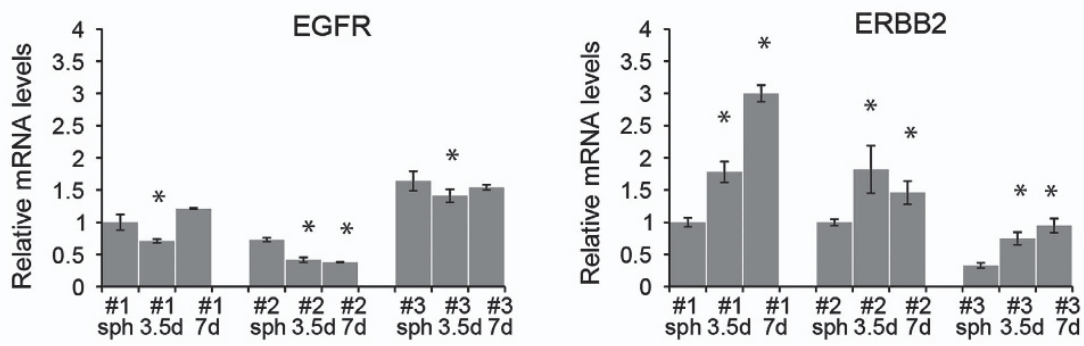

d

BCSC

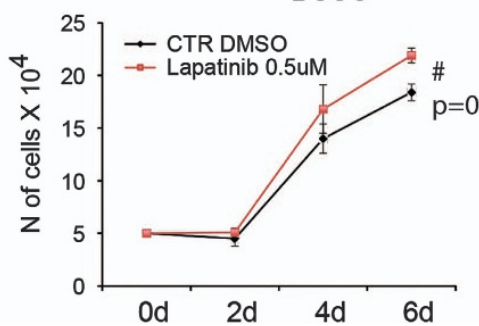

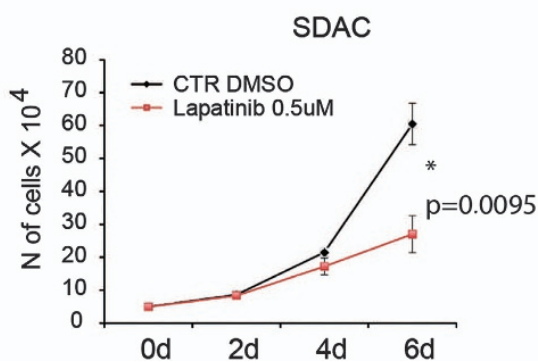

Figure 1 ERBB2 and EGFR expression pattern in BCSCs and during differentiation. (a) Immunohistochemistry of three different paraffin-embedded primary tumor tissues (left), CSCs lines derived from tumors (centre) and SDACs (right) stained with anti-HER2 antibodies (pink-brown) (Scale bar: $20 \mu \mathrm{M}$ ). ERBB2 is expressed in primary tumors but not in CSCs lines and starts to be re-expressed under differentiating condition (SDAC). (b) Western blot analysis of BCSC lines (BCSC\#1, BCSC\#2 and BCSC\#3) and stem cells under differentiating condition (SDAC) for 3.5 and 7 days using antibodies against ERBB2, EGFR and Actin as a loading control. The corresponding molecular weights are indicated on the left (KDa). ERBB2 and EGFR expression increases during differentiating condition. (c) qRT-PCR of ERBB2 and EGFR levels of BCSC lines (BCSC\#1, BCSC\#2 and BCSC\#3) and SDAC of the same lines differentiated for 3.5 and 7 days. Data represent mean \pm S.D. of three different experiments analyzed in triplicate. Statistical significance was analyzed using Student's $T$ test $\left({ }^{*} P<0.05\right)$. (d) BCSCs are resistant to Lapatinib treatment. Cell proliferation assay of BCSCs and SDAC untreated (ctr) or treated with $0.5 \mu \mathrm{M}$ of Lapatinib at the indicated time points (days). SDAC were differentiated for 3.5 days and then plated for growth curve analysis. BCSCs express low receptors levels and are more resistant to treatment than SDAC. Data represent mean \pm S.D. of three different experiments and $P$ values are shown in the graphs 

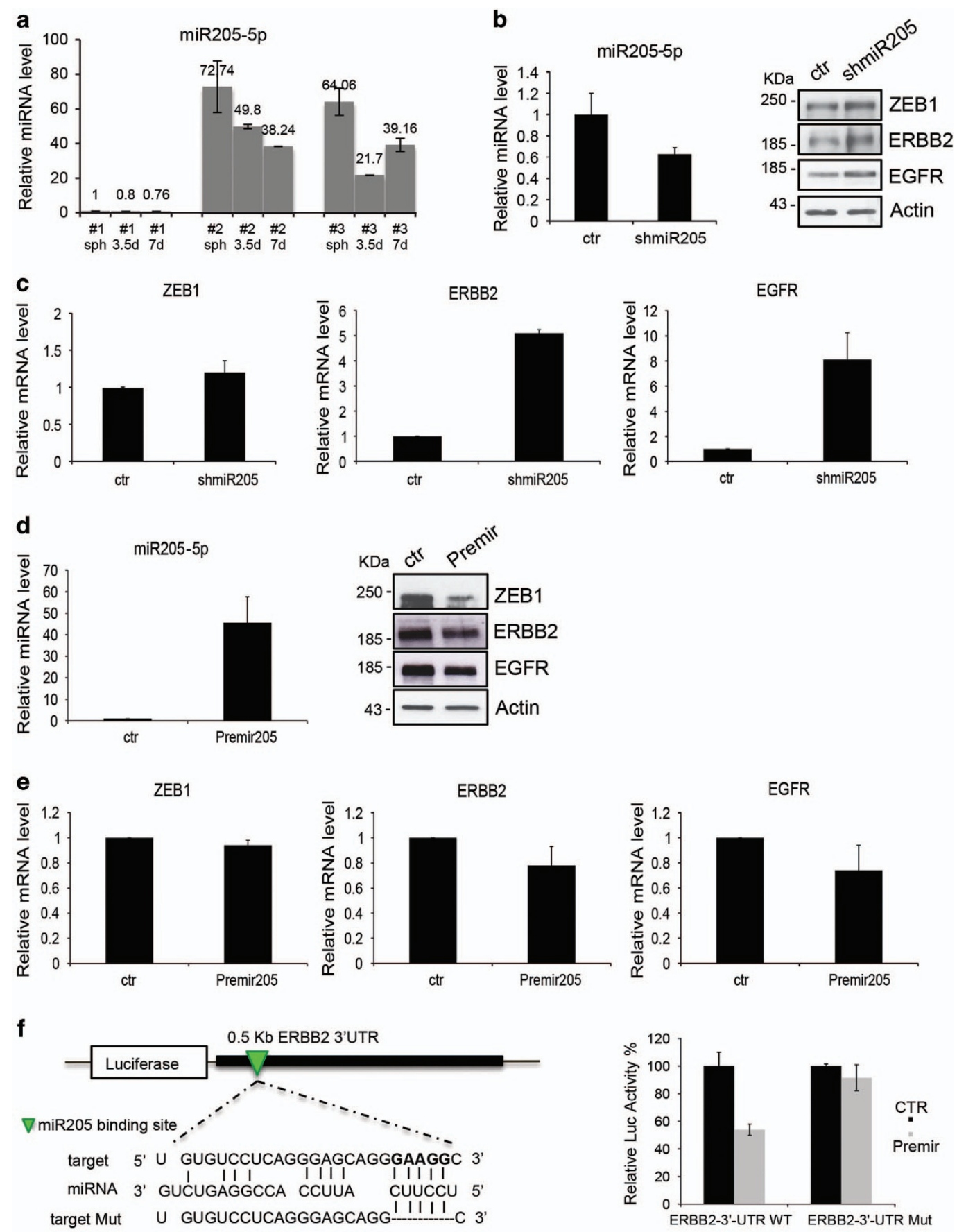

g
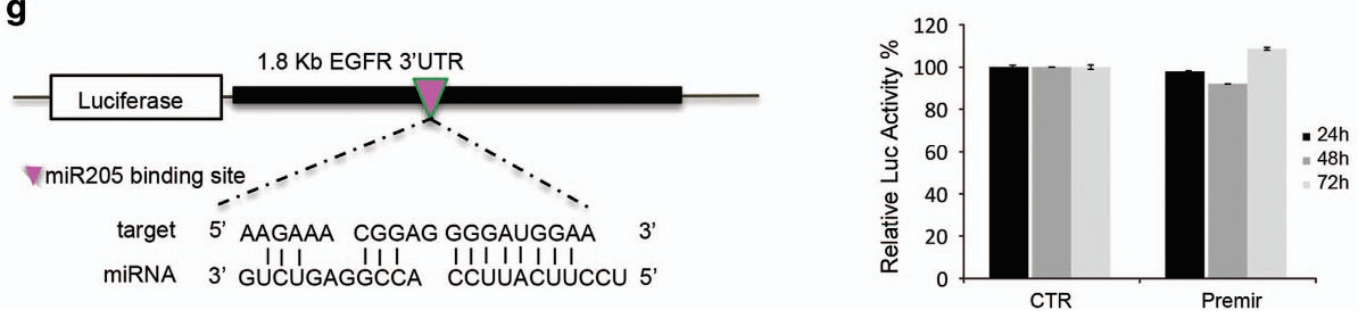
we studied the role of p63 in this pathway, because it has been reported to promote the transcription of EGFR. ${ }^{30}$ Interestingly, it has also been shown that p63 controls miR-205-5p expression. ${ }^{18,19}$

To evaluate the potential role of p63 in miR-205-5p -ERBB family axis, we first checked the expression levels of p63 in our model. As shown in Figure 3, all three lines tested express detectable mRNA (Figure $3 a$ ) and protein (Figure $3 b$ ) levels of p63. In addition, we tested whether p63 was also able to drive the expression of EGFR in BCSCs as previously reported. As shown in Figure 3c, silencing of 163 in BCSC \#1 cells results in reduced expression of EGFR, whereas upregulation of the $\Delta \mathrm{N}$ but not the TA isoform results in increased EGFR levels (Figure 3d). Interestingly, p63 levels increase in more differentiated cancer cells as compared with cells grown as spheroids in parallel with the reduction of miR-205-5p expression (Figure $3 e$ ). We therefore investigated whether miR-205-5p is capable of regulating p63 expression. As shown in Figure 4a, miR-205-5p knockdown results in a significant p63 upregulation both at the mRNA and protein levels. Consistently, miR-205-5p overexpression results in reduced p63 levels (Figure 4b). Bio-informatic analysis of p63 $3^{\prime}$-UTR identified a putative miR-205-5p seed region (Figure 4c, left). We therefore cloned the p63 3'-UTR containing the miR-205-5p binding site into the pGL3 control vector downstream the luciferase gene. Co-transfection of premiR-205 and the WT p63 3'-UTR construct in SKBR3 cells resulted in significant inhibition of luciferase activity compared with the cells in which the WT p63 3'-UTR construct was co-transfected with a control vector (Figure 4c, right). These data were confirmed by mutation of $m i R-205-5 p$ binding site within the p63 3'-UTR (p63-3'-UTR Mut) that abolishes the ability of $m i R-205-5 p$ to regulate the luciferase expression leading to an increase of luciferase activity. We therefore believe that miR-205-5p regulates expression of EGFR through regulation of $\Delta$ Np63. Interestingly, we confirm that as reported in literature, p63 regulates miR-205-5p expression thus creating a regulatory feedback loop. In fact, p63 silencing

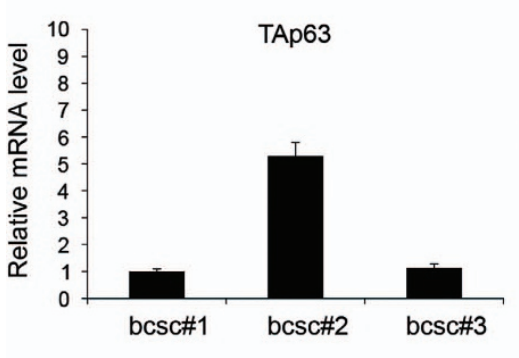

C

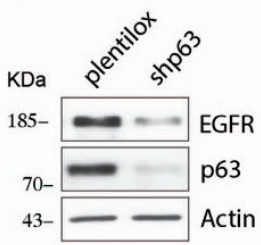

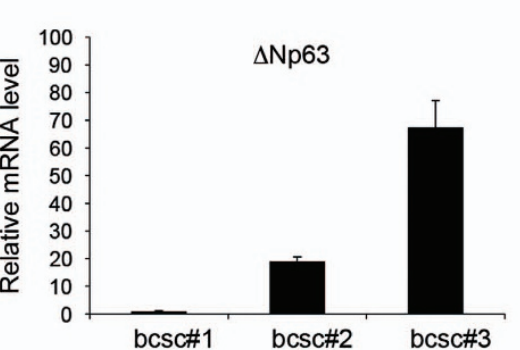

b

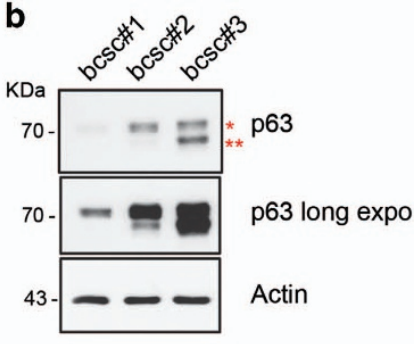

d

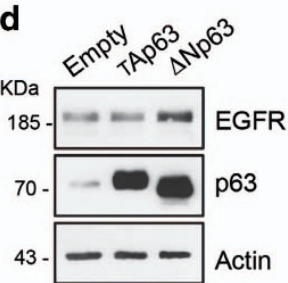

e

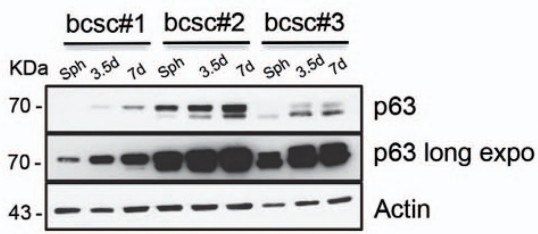

Figure 3 p63 expression pattern in BCSCs and during differentiation. (a) p63 expression pattern in BCSCs. TAp63 (left) and $\triangle$ Np63 (right) expression levels in BCSC\#1, BCSC\#2 and BCSC\#3 were assessed by qRT-PCR. All three stem cell lines tested show p63 detectable levels. (b) Western blot analysis of p63 protein levels in BCSC\#1, BCSC\#2 and BCSC\#3 normalized with Actin levels. Numbers on the left indicate molecular weight (KDa) and symbol on the right indicate * Tap63 and ** $\Delta$ Np63, respectively. (c) EGFR protein levels in BCSC\#1 infected with p63 silencing (shp63) lentivector. p63 silencing leads to EGFR downregulation. (d) $\triangle$ Np63 and not Tap63 regulates EGFR expression. Western blot of EGFR protein levels in BCSC\#1 infected with TAp63 or $\triangle$ Np63 overexpression lentivector or a control vector (Empty). (e) p63 expression pattern in BCSC\#1, BCSC\#2 and BCSC\#3 and SDAC collected at 3.5 and 7 differentiation days. Western blot of p63 levels shows an increase of both TAp63 and $\triangle$ Np63 isoforms during differentiating condition (middle: long exposure to better evaluate p63 expression in all three BCSC lines)

Figure 2 miR-205-5p regulates ERBB receptors expression in BCSCs. (a) qRT-PCR quantification of miR-205-5p expression in BCSC\#1, BCSC\#2 and BCSC\#3 and SDAC differentiated for 3.5 and 7 days. miR-205-5p is downregulated during differentiation in all three stem cell lines tested. Data represent mean \pm S.D. of three different experiments analyzed in triplicate. (b and c) miR-205-5p regulates ERBB2 and EGFR expression. QRT-PCR and western blotting analysis of EGFR and ERBB2 expression levels in BCSC\#1 infected with miR-205-5p silencing lentivector (shmiR-205-5p). miR-205 knockdown results in EGFR and ErbB2 upregulation both at the mRNA and protein levels. ZEB-1, a wellestablished miR-205 target, was used as a control to further confirm functional miR-205 silencing. (d and e) miR-205-5p overexpression results in ERBB receptors downregulation. qRT-PCR and western blot analysis of EGFR, ERBB2 and Zeb-1 expression levels in BCSC\#1 infected with PremiR-205 lentivector. All qRT-PCR data represent mean \pm S.D. of three different experiments analyzed in triplicate. (f) miR-205-5p directly targets ERBB2 at 3'-UTR. Schematic model of the predicted ERBB2 3'-UTR binding site for miR-205-5p and alignment of the seed region with both wild-type and mutated ERBB2 3'-UTR (left). On the right, relative luciferase activity is shown. SKBR-3 cells were cotransfected for $24 \mathrm{~h}$ with pGL3-ERBB2 3'-UTR luciferase construct (WT or Mut 3'-UTR), premiR 205 construct or a control vector (CTR). The results represent mean \pm S.D. of three different experiments analyzed in triplicate. (g) EGFR is not a direct target of miR-205-5p. Representation of the interaction between miR-205-5p and the putative binding 

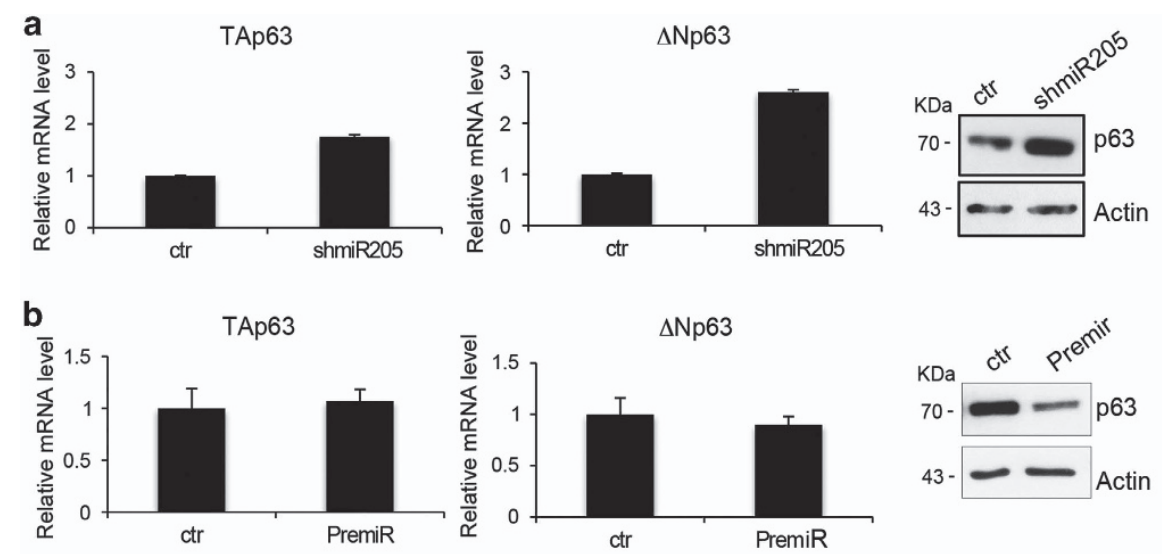

C
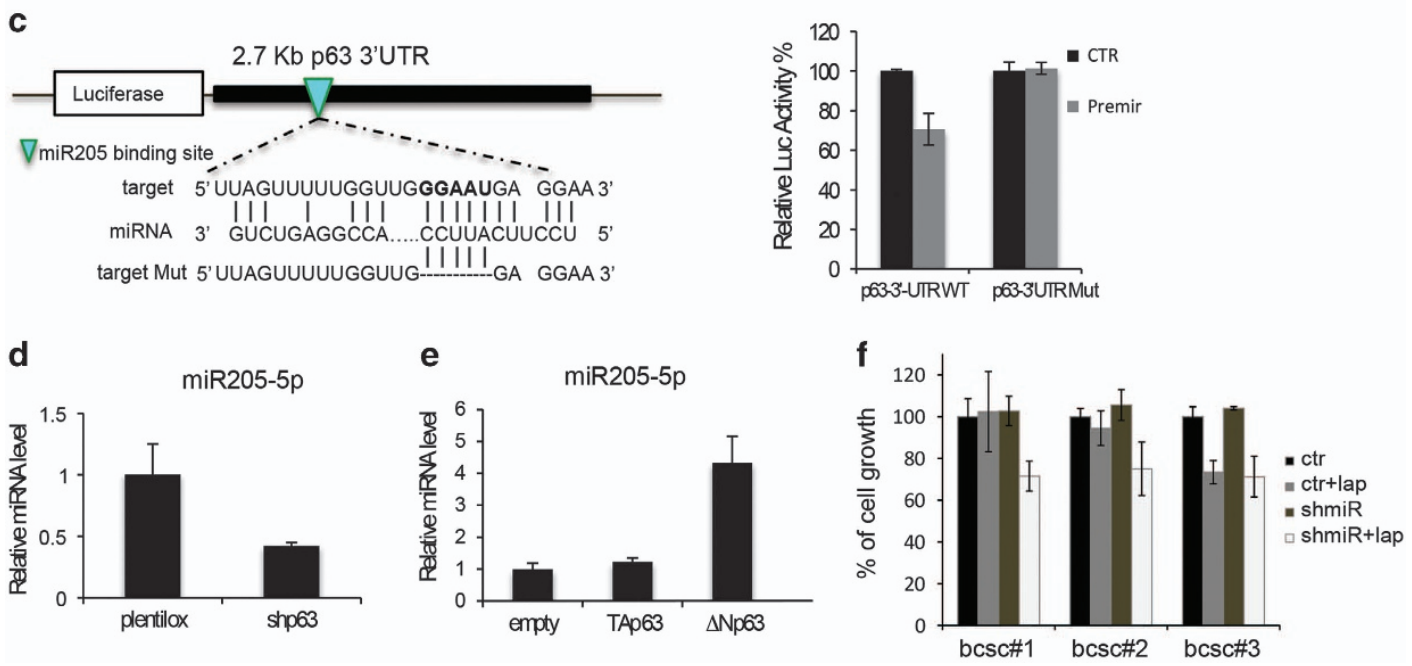

Figure 4 miR-205-5p regulates p63 expression in BCSCs. (a) miR-205-5p regulates p63 levels. qRT-PCR (left) and western blotting analysis (right) of p63 expression levels in BCSC\#1 infected with miR-205-5p silencing lentivector (shmiR-205-5p). (b) qRT-PCR (left) and western blotting (right) of p63 expression levels in BCSC\#1 infected with Premir205 lentivector. miR-205-5p overexpression results in p63 downregulation mainly at protein levels. (c) miR-205-5p directly targets p63-3'-UTR. On the left, putative miR-205-5p binding site on p63 wild-type 3'-UTR and alignment of the seed sequence with both WT and mutated p63 3'-UTR. On the right, SKBR-3 cells were co-transfected for $48 \mathrm{~h}$ with pGL3-p63 3'-UTR luciferase construct (WT or Mut 3'-UTR), premiR 205 construct or a control vector (CTR). Cloning p63 3'-UTR WT, and not the mutated one, into a luciferase reporter gene leads to diminished luciferase activity in the presence of Premir-205. (d) p63 regulates miR-205-5p expression. qRT-PCR of miR-205-5p expression levels in BCSC\#1 infected with shp63 lentivector (shp63) or a control vector (plentilox). (e) qRT-PCR of miR-205-5p expression levels upon Tap63 or $\triangle$ Np63 overexpression in BCSC\#1. $\triangle$ Np63 overexpression results in miR-205-5p upregulation. (f) miR-205-5p downregulation re-sensitize BCSCs to Lapatinib treatment. Percentage of cell growth of BCSC\#1, BCSC\#2 and BCSC\#3 infected with shmiR-205-5p lentivector (shmiR-205-5p) or a control vector (CTR) and treated or untreated with $0.5 \mu \mathrm{M}$ Lapatinib for 6 days

results in reduced miR-205-5p levels (Figure 4d), whereas upregulation of the $\Delta \mathrm{N}$ but not the TA isoform results in significant increase of miR-205-5p levels (Figure 4e).

We therefore sought out to re-sensitize BCSCs to Lapatinib treatment downregulating mir-205-5p, that we showed is responsible for reduced expression of EGFR and ErbB2 in these cells. As shown in Figure 4f, silencing miR-205-5p in BCSCs and treating them with Lapatinib strongly reduces cell proliferation, sensitizing cells to anti-Her2/EGFR treatments. These findings indicate that miR-205-5p is able to regulate ErbB receptors expression thus leading to targeted therapy resistance of BCSCs.

\section{Discussion}

Growing evidence suggest that a subset of cells within the tumor, refered as CSCs are capable of escaping anti-cancer treatment driving tumor progression, metastasis and relapse. ${ }^{1}$
Many studies have therefore focused on the identification of pathways that are essential in determining the CSC phenotype. It is well known that miRNAs contribute to carcinogenesis and tumor development acting as oncogenes or as tumor suppressors depending on specific targets and tumor microenvironment. Altered miR-205-5p expression has been involved in several types of solid tumors and, to date, its target include tumor suppressors like PTEN ${ }^{12}$ and SHIP2, ${ }^{31}$ oncogenes such as HER3 ${ }^{32} \mathrm{PKC} \varepsilon,{ }^{17}$ pro-metastatic factors Zeb1 and Zeb-2, ${ }^{14}$ and the angiogenic gene VEGFA. ${ }^{32}$ Moreover, miR-205-5p has been shown to be essential for mouse development, ${ }^{33,11}$ particularly for the expansion of progenitor and stem cell populations in epidermis, hair follicles and more importantly in mammary gland during neonatal development; ${ }^{33,12}$ therefore, we hypothesize its mis-regulation could be translated in maintaining the CSC phenotype. In breast cancer, miR-205-5p was found to be either up- or downregulated compared with normal tissue, but its 
expression in BCSCs remains still unknown. Here, we show that miR-205-5p is highly expressed in human BCSCs compared with more differentiated tumor cells and that it directly targets ERBB receptors leading to their downregulation. In fact while it was known that ERBB3 is a direct target of miR-205-5p, we show that it also regulates ErbB2 and EGFR. While ErbB2 appears to be a direct target of this miR, EGFR regulation is mediated through the regulation of $p 63$ that has already been shown to be able of regulating transcription of EGFR. ${ }^{30}$ Indeed, here we show for the first time that p63 is a direct target of miR-205-5p and confirm previous reports showing that in turn $\Delta \mathrm{Np63}$ regulates miR-205-5p expression. ${ }^{19,18}$ These data show that, therefore, there is a feedback loop finely regulating expression of $m i R-205-5 p$ and p63 that have a role in determining some of the phenotypic features of BCSCs including surface expression of ERBB receptors.

Intriguingly, we show that low expression of ERBB receptor family members in BCSCs contributes to resistance of these cells to agents such as Lapatinib used in breast cancer therapy. Survival of these cells could then lead to tumor progression and suggests that miR-205-5p could be an important target to improve outcome of patients with Her2overexpressing breast cancer.

In conclusion, we want to point out that we identified for the first time two new direct targets of $m i R-205-5 p$ and shown that this miRNA has an important role in determining BCSCs phenotype and contributes to their resistance to targeted therapy.

\section{Materials and Methods}

BCSC isolation and culture. BCSCs were isolated from human breast cancer tissues obtained from patients as previously described ${ }^{34}$ and were cultured in a selective medium ${ }^{34}$ supplemented with $10 \mathrm{ng} / \mathrm{ml} \beta F G F$ (Peprotech, London, UK), $20 \mathrm{ng} / \mathrm{ml}$ EGF (Peprotech) to a final concentration $5 \times 10^{4} / \mathrm{ml}$ in ultra low attachment flask (Corning, New York, NY, USA) at $37^{\circ} \mathrm{C}$ in a $5 \%(\mathrm{v} / \mathrm{v}) \mathrm{CO}_{2}$ humidified chamber. BCSCs were induced to differentiate in order to obtain SDACs by culturing them in adherent condition in D-MEM with high glucose (Euroclone, Milan, Italy) supplemented with $10 \%$ (v/v) fetal bovine serum (Euroclone). The tumor s were histopathologically classified as follows: BCSC\#1 is an invasive ductal carcinoma, grading G2, ER 90\%, PR 60\%, HER2/neu 3+ and ki67 >10\%; BCSC\#2 is an invasive ductal carcinoma, grading G2, ER $90 \%$, PR $60 \%$, HER2/neu 3+ and ki67 25\%, BCSC\#3 is an invasive ductal carcinoma, grading G2, ER $80 \%$, PR $80 \%$, HER2/neu $3+$ and ki67 $>10 \%$. HER2 status has been assigned according to the FDA guidelines.

Constructs. To generate miR-205-5p expression lentiviral vector, a $326 \mathrm{bp}$ fragment carrying pre-miR-205 was amplified from $\mathrm{H} 1299$ genomic DNA by the Platinum Taq polymerase high fidelity (Life Technologies, Waltham, MA, USA) using PCR primers: miR-205 F 5'-cggctagccgaggtccttgacatct-3' and miR-205 R $5^{\prime}$-ccctcg agggcctaagtcagagtta-3'.

The amplified fragment was cloned into pLenti-CMV-RFP-2A-Puro (Applied Biologicals Marerials Inc., Richmond, BC, Canada) lentiviral vector at Nhe-Xhol sites.

To generate shRNA miR-205 lenti-vector, we cloned miR-205-5p into pSIH-H1copGFP Vector (System Biosciences, Mountain View, CA, USA), at EcoRI-BamHI sites, using PCR oligonucleotides:

shmiR-205 $15^{\prime}$-gatcctccttcattccaccggagtctgcttcctgtcagacagactccggtggaatgaagga 'ttttg-3' shmiR-205 2 5'-aattcaaaaatccttcattccaccggagtctgtctgacaggaagcagactccg gtggaatgaaggag- $3^{\prime}$.

To generate a luciferase reporter carrying the ErbB2-3'-UTR with a putative miR-205-5p binding site, we amplified a 512 bp fragment by PCR from the first nucleotide after the stop codon to the last nucleotide before the polyadenylation signal from human genomic DNA using the following primers: ERBB2-UTR SpeF $5^{\prime}$-gactagtcaccagaaggccaagtccg- $3^{\prime}$ and ERBB2-UTR SpeR 5'-ggactagtcctcatc tttaaaaaaacaaaac-3'. The fragment, after Spel restriction, was ligated to a compatible Xbal linearized pGL3 Control vector (Promega, Madison, WI, USA). The miR-205-5p predicted target site ( $5 \mathrm{bp}, \mathrm{GAAGG}$ ) was deleted by PCR using the following primers: Her2MutFw 5'- gccctgatgtgtcctcagggagcaggcc-3' and Her2MutRev $5^{\prime}$-tgatgccagc agaagtcaggcctgctcc-3' with QuikChange Lightning Site-Directed Mutagenesis kit (Agilent Technologies, Santa Clara, CA, USA).

The same procedure was used to clone 2770 bp p63 3'-UTR fragment in a pGL3 Control vector using the following primers: p63UTR-SpelF 5'-ggccactagtgcctcaccatgtgagctcttc-3'; p63UTR-SpelR $5^{\prime}$-ggccactagtgcatgtcctggcaaacaaaaagag-3' as previously described. ${ }^{35}$ Mutation ( $5 \mathrm{bp}$, GGAAU) was introduced into the miRNA binding site with QuikChange Lightning Site-Directed Mutagenesis kit (Agilent Technologies) using the following primers: Fwp63Mut gttttggttggaggaaaattctt aaaaggcccatagcagc

Revp63Mut gctgctatgggccttttaagaatttcctccaaccaaaaac.

EGFR 3'-UTR WT luciferase reporter vector was kindly provided by CM Croce (Ohio State University Wexner Medical Center and Comprehensive Cancer Center, Columbus, $\mathrm{OH}, \mathrm{USA}$ )

To generate TAp63 $\alpha$-Tween and $\triangle N p 63 \alpha$-Tween expression lentiviral vectors, we subcloned inserts from TAp63 $\alpha-p c D N A$ and $\triangle N p 63 \alpha-p c D N A$ constructs, kindly provided by Prof. G Melino, ${ }^{36}$ into Xbal-Xhol unique sites of Tween lentiviral vector ${ }^{37}$ under the control of $\mathrm{hCMV}$ promoter. This vector constitutively expresses GFP under the control of hPGK promoter.

For shp63 construct, to downregulate p63 expression, the pLL3.7-p63-7.2 was generated by insertion in pLL3.7 vector (Addgene plasmid 11795, Addgene, Cambridge, MA, USA) of oligos targeting the following sequence: GAGTGGAATGAC TTCAACTTT. ${ }^{38}$

The cloning was performed according to the pLL3.7 protocol from the Tyler Jacks laboratory at MIT (https://www.addgene.org/static/data/94/67/16242780-af64-11e090fe-003048dd6500.pdf).

A CMV-EGFP reporter cassette is included in the vector to monitor expression.

All PCR products were verified by DNA sequencing.

Infections. Lentiviruses were produced by transient cotransfection of a threeplasmid expression system in the packaging 293T cells, using the calcium phosphate transfection kit (Invitrogen, Life Technologies). Cells were incubated for $7 \mathrm{~h}$ with the transfection reagents and viral supernatant was collected $48 \mathrm{~h}$ after transfection and filtered through $0.45 \mu \mathrm{m}$ pore vacuum sterile filtration system (Millipore, Life Science, Darmstadt, Germany). Then, BCSCs were plated in a sixwell ultra-low attachment plate (Corning) with viral supernatant and $4 \mu \mathrm{g} / \mathrm{ml}$ of polybrene. Plates were centrifuged for $45 \mathrm{~min}$ at 1800 r.p.m. and incubated at $37^{\circ} \mathrm{C}$ for $75 \mathrm{~min}$ in a $5 \% \mathrm{CO}_{2}$ humified chamber. Cells were then washed twice and replated in fresh medium. ${ }^{39}$ Infection efficiency was assessed by flow cytometry (FACSCanto II Instrument, BD Biosciences, San Jose, CA, USA) $48 \mathrm{~h}$ post-infection evaluating the percentage of GFP-positive or RFP-positive cells measured. Data were analyzed with CELLQuest software (BD Biosciences).

Quantitative reverse transcription-PCR. For miRNA detection, RNA was extracted from BCSCs by using miRVana miRNA Isolation kit (Ambion by Life Technologies). A total of $50 \mathrm{ng}$ of RNA was used for reverse transcription using TaqMan MicroRNA Reverse Transcription Kit (Applied Biosystem by Life Technologies) with the following stem loop specific primers: miR-205-5p RT: $5^{\prime}$-gttgg ctctggtgcagggtccgaggtattcgcaccagagccaaccagact-3' and U44-RT: 5'-gttggctctggt gcagggtccgaggtattcgcaccagagccaacagtcagtt-3'.

Real-time PCR was performed by using FastStart Universal Probe Master (Rox) (Roche, Basel, Switzerland) and Universal Probe Library, Probe \#21 (Roche) using the following primers: miR-205-5p Fw 5'-gcggcggtgtagtgttccta-3' and universal Reverse primer: $5^{\prime}$-gtgcagggtccgaggt-3'. miR-205-5p expression was calculated relative to U44 rRNA with the following primer: $5^{\prime}$-gcggcggcctggatgatgatag- 3 ' with an amplification protocol as follows: one cycle of $95^{\circ} \mathrm{C}$ for $10 \mathrm{~min}$ and 40 cycles of $95^{\circ} \mathrm{C}$ for $15 \mathrm{~s}$ and $60^{\circ} \mathrm{C}$ for $1 \mathrm{~min}$ on an Applied Biosystem 7900HT Sequence Detection System (Applied Biosystems, Waltham, MA, USA). Relative quantification of miRNA expression was calculated according to the comparative method of $2^{-}{ }^{\Delta \Delta C T}$.

For detection of other genes, a total of $500 \mathrm{ng}$ of RNA was used for reverse transcription using TaqMan Reverse Transcription Reagents (Applied Bioisystem, Life Technologies) and Real-time PCR was performed by using the Platinum SYBR Green qPCR SuperMix UDG with Rox (Invitrogen, Life Technologies), with an amplification program as follows: one cycle of $95^{\circ} \mathrm{C}$ for $3 \mathrm{~min}$ and 40 cycles of $95^{\circ} \mathrm{C}$ for $20 \mathrm{~s}$ and $60^{\circ} \mathrm{C}$ for $1 \mathrm{~min}$. The reaction was followed by a melting curve protocol according to the specification of the ABI $7900 \mathrm{HT}$ instrument (Applied Biosystems). Primers used 
were as follows: Tap63 (NM_003722.4) F: 5'-ttgagattagcatggactgtatcc-3'and R: 5'-gttctgaatctgctggtccat-3'; $\Delta$ Np63 (NM_001114980.1) F: 5'-ggttggcaaaatcctggag-3' and R: $5^{\prime}$-ggttcgtgtactgtggctca-3'. For ErbB2 (NM_004448.2) F: 5'-gggaaacct ggaactcacct-3' and R: 5'-ccctgcacctcctggata-3'; for EGFR (NM_005228.3) F: 5'-ttcc tcccagtgcctgaa-3' and R: 5'-gggttcagaggctgattgtg-3'; for ZEB1 F: 5'-gcacaaccaag tgcagaaga- $3^{\prime}$ and R: $5^{\prime}$-gcctggttcaggagaagatg- $3^{\prime}$.

All genes expression were normalized using human $\beta$-actin as housekeeping gene, and primers used were ActF $5^{\prime}$-cagctcaccatggatgatgatatc- $3^{\prime}$ and ActR $5^{\prime}$-aagccggccttgcacat $-3^{\prime}$. Relative quantification of gene expression was calculated according to the comparative method of $2^{-\Delta \Delta C T}$.

Microarray. Total RNA was extracted from BCSC \#1 BCSCs and from BCSC \#1 differentiated cells at 7 days, according to Trizol protocol (Ambion by Life Technologies). Total RNA was used for miRNA microarray analysis (G4470B, Agilent Technologies). This chip allows the simultaneous analysis of 723 human miRNAs (miRBase release 10.1). RNA labeling and hybridization were performed in accordance to the manufacturer's indications. Agilent scanner and the Feature Extraction 10.5 software (Agilent Technologies) were used to obtain the microarray raw data. Microarray results were analyzed using the GeneSpring GX 12 software (Agilent Technologies). Data transformation was applied to set all the negative raw values at 1.0, followed by Quantile normalization and log2 transformation. Differentially expressed miRNAs were identified by using a moderated $t$-test and Benjamini-Hochberg correction (adjusted $P<0.05$ ). Differentially expressed genes were used in Cluster Analysis, using the Pearson correlation as a measure of similarity.

Western blotting. Proteins were extracted with a lysis buffer (TRIS-HCl 50 $\mathrm{mM} \mathrm{pH}$ 8, NaCl 150 mM, Triton X-100 1\%, NaF 100 mM, EDTA 1 mM, MgCl 1 mM, Glycerol 10\%) containing a protease inhibitor cocktail (Sigma-Aldrich, St. Louis, MO, USA) and a phosphatase inhibitor cocktail (Roche) as previously described. ${ }^{40}$ Equal amounts of total protein were subjected to SDS-PAGE and then electrotransferred to nitrocellulose membranes. The membranes were blocked with $5 \%$ non-fat dry milk in PBS with $0,1 \%$ Tween 20 and incubated overnight using the following antibodies: anti $\beta$-Actin A5441 (Sigma-Aldrich), anti-EGF Receptor (D38B1) XP Rabbit mAb (Cell Signaling, Danvers, MA, USA), anti-ErbB2 (D8F12) XP Rabbit mAb (Cell Signaling) anti-ZEB1 (Millipore, Life Science), anti-p63 Y4A3 (Sigma-Aldrich) or anti-p63 alpha (D2K8X) XP Rabbit mAb (Cell Signaling). After wash, membranes were hybridized with horseradish peroxidase-conjugated secondary antibodies (rabbit and mouse, Bio-Rad, Hercules, CA, USA). Detection was performed with Plus-ECL chemiluminescence kit (PerkinElmer, Inc., Waltham, MA, USA) or with SuperSignal West Dura extended duration substrate kit (Thermo Scientific, Waltham, MA, USA)

Immunohistochemistry. BCSCs and SDACs derived from BCSC\#1, BCSC\#2 and BCSC\#3 lines, were spotted on microscope slides with cytospin at 900 r.p.m. for $3 \mathrm{~min}$ and then were fixed in formalin $10 \%$ neutral buffered for $15 \mathrm{~min}$. BCSC, SDAC and paraffin-embedded primary tumor tissues were stained using rabbit polyclonal (HercepTest, Dako, Glostrup, Denmark) antibodies following the manufacturer's instructions. Antigen-antibody reaction was visualized using an antirabbit \#K4003 polymer-based detection system (EnVision Kit, Dako), and using diaminobenzidine as the chromogen. In control sections, the specific primary antibody was replaced with rabbit non immune serum. Primary tumors BCSC \#1, BCSC\#2 and BCSC\#3 were scored according to HercepTest (HT) (Dako) and classified as HT $3+{ }^{41}$

Luciferase assay. Human breast carcinoma cell line (SKB-R3) was grown in McCoy's 5A Medium (Euroclone, Milan, Italy) supplemented with $10 \%(\mathrm{v} / \mathrm{v})$ fetal bovine serum (Euroclone). A total of $8 \times 10^{4}$ SKB-R3 cells were seeded in 12-well dishes $24 \mathrm{~h}$ before transfection. Three hundred and twenty-five nanograms of pGL3 vectors, $650 \mathrm{ng}$ of pLenti-CMV-RFP-2 A-Puro vectors and $10 \mathrm{ng}$ of Renilla luciferase vector were co-transfected using Lipofectamine 2000 (Invitrogen by Life Technologies). Luciferase activities of cellular extracts were measured 24 and $48 \mathrm{~h}$ after transfection for ErbB2 and p63 3'-UTR, respectively, and for 24, 48 and $72 \mathrm{~h}$ after transfection for EGFR 3'-UTR, by using a Dual-Glo-Luciferase Reporter Assay System (Promega, Fitchburg, WI, USA) with a Lumat LB 9507 luminometer. Efficiency of transfection was normalized using Renilla luciferase activity.

FACS analysis. For flow cytometric analysis of EGFR and ErbB2 surface markers, $20 \times 10^{4}$ cells per sample, for all three BCSCs and SDACs tested
(BCSC\#1, BCSC\#2 and BCSC\#3), were washed in PBS, resuspended in $100 \mu \mathrm{l}$ of specific antibody diluted in $0.5 \% \mathrm{BSA}$, and incubated for $20 \mathrm{~min}$ at room temperature. For EGFR and ErbB2 staining $10 \mu \mathrm{g} / \mathrm{ml}$ Trastuzumab (Roche) and Cetuximab (Merck Serono, Darmstadt, Germany) were used, respectively. Then, samples were incubated for $20 \mathrm{~min}$ in the dark with secondary fluorescent antihuman-R-PE-conjugated antibody (H10104, Life Technologies, $1: 300$ in $0.5 \%$ BSA). Cell viability solution (555815, BD Biosciences) was used for detection of non-viable cells according to the manufacturer's protocol. Samples were then washed and stored at $4^{\circ} \mathrm{C}$ in the dark until acquisition.

A FACSCantoll flow cytometer, running with FACSDiVa software (BD Biosciences), was used for sample acquisition and analysis.

Cell proliferation assay. BCSCs, SDACs and BCSCs infected with shmiR-205-5p were seeded into six-well plate at $5 \times 10^{4}$ cells per well. Viable cell count was performed with Trypan Blue reagent (Sigma-Aldrich) at the indicated time points. When indicated, cells were treated with $0.5 \mu \mathrm{M}$ Lapatinib (Biovision, Milpitas, CA, USA).

Bioinformatics. miR-205-5p target sites on p63 3'-UTR, ERBB2 3'-UTR and EGFR 3'-UTR were predicted by RNA Hybrid software available at http://bibiserv. techfak.uni-bielefeld.de/rnahybrid/submission.html

\section{Conflict of Interest}

The authors declare no conflict of interest.

Acknowledgements. This work was supported by grants from AIRC (2011-IG11450) and Italian Ministry of Health (Ricerca Finalizzata 2009, RF-20091491608) to VDL and by Italian Ministry of Research and University FIRB project RBAP11BYNP 002 to MN

1. Liu S, Clouthier SG, Wicha MS. Role of microRNAs in the regulation of breast cancer stem cells. J Mammary Gland Biol Neoplasia 2012; 17: 15-21.

2. Spielmann M, Roché H, Delozier T, Canon J-L, Romieu G, Bourgeois $H$ et al. Trastuzumab for patients with axillary-node-positive breast cancer: results of the FNCLCC-PACS 04 trial. I Clin Oncol 2009; 27: 6129-6134.

3. Goss PE, Smith IE, O'Shaughnessy J, Ejlertsen B, Kaufmann M, Boyle F et al. Adjuvant lapatinib for women with early-stage HER2-positive breast cancer: a randomised, controlled phase 3 trial. Lancet Oncol 2013; 14: 88-96.

4. Arteaga CL, Sliwkowski MX, Osborne CK, Perez E a, Puglisi F, Gianni L. Treatment of HER2-positive breast cancer: current status and future perspectives. Nat Rev Clin Oncol 2012; 9: 16-32.

5. Garrett JT, Arteaga CL. Resistance to HER2-directed antibodies and tyrosine kinase inhibitors: Mechanisms and clinical implications. Cancer Biol Ther 2011; 11: 793-800.

6. Lewis BP, Burge CB, Bartel DP. Conserved seed pairing, often flanked by adenosines, indicates that thousands of human genes are microRNA targets. Cell 2005; 120 15-20

7. Bagga S, Bracht J, Hunter S, Massirer K, Holtz J, Eachus R et al. Regulation by let-7 and lin-4 miRNAs results in target mRNA degradation. Cell 2005; 122: 553-563.

8. Olsen PH, Ambros V. The lin-4 regulatory RNA controls developmental timing in Caenorhabditis elegans by blocking LIN-14 protein synthesis after the initiation of translation. Dev Biol 1999; 216: 671-680.

9. Calin GA, Croce CM. MicroRNA signatures in human cancers. Nat Rev Cancer 2006; 6 : 857-866.

10. Calin GA, Konopleva M. Small gene, big number, many effects. Blood 2012; 120: 240-241.

11. Farmer DT, Shariat N, Park CY, Liu HJ, Mavropoulos A, McManus MT. Partially penetrant postnatal lethality of an epithelial specific MicroRNA in a mouse knockout. PLoS One 2013; 8. e76634

12. Greene SB, Gunaratne PH, Hammond SM, Rosen JM. A putative role for microRNA-205 in mammary epithelial cell progenitors. J Cell Sci 2010; 123: 606-618.

13. Wu H, Zhu S, Mo Y-Y. Suppression of cell growth and invasion by miR-205 in breast cancer. Cell Res 2009; 19: 439-448.

14. Gregory PA, Bert AG, Paterson EL, Barry SC, Tsykin A, Farshid G et al. The miR-200 family and miR-205 regulate epithelial to mesenchymal transition by targeting ZEB1 and SIP1. Nat Cell Biol 2008; 10: 593-601.

15. Matsushima $\mathrm{K}$, Isomoto $\mathrm{H}$, Yamaguchi $\mathrm{N}$, Inoue $\mathrm{N}$, Machida $\mathrm{H}$, Nakayama $\mathrm{T}$ et al. MiRNA-205 modulates cellular invasion and migration via regulating zinc finger E-box binding homeobox 2 expression in esophageal squamous cell carcinoma cells. J Trans/ Med 2011; 9: 30.

16. Xie H, Zhao Y, Caramuta S, Larsson C, Lui WO. miR-205 expression promotes cell proliferation and migration of human cervical cancer cells. PLOS One 2012; 7 e46990. 
17. Gandellini P, Folini M, Longoni N, Pennati M, Binda M, Colecchia M et al. miR-205 Exerts tumor-suppressive functions in human prostate through down-regulation of protein kinase Cepsilon. Cancer Res 2009; 69: 2287-2295.

18. Tran MN, Choi W, Wszolek MF, Navai N, Lee I-LC, Nitti G et al. The p63 protein isoform $\Delta \mathrm{Np63 \alpha}$ inhibits epithelial-mesenchymal transition in human bladder cancer cells: role of MIR-205. J Biol Chem 2013; 288: 3275-3288.

19. Tucci P, Agostini M, Grespi F, Markert EK, Terrinoni A, Vousden KH et al. Loss of p63 and its microRNA-205 target results in enhanced cell migration and metastasis in prostate cancer. Proc Natl Acad Sci USA 2012; 109: 15312-15317.

20. Allocati N, Di llio C, De Laurenzi V. P63/P73 in the control of cell cycle and cell death. Exp Cell Res 2012; 318: 1285-1290.

21. Graziano V, De Laurenzi V. Role of p63 in cancer development. Biochim Biophys Acta 2011; 1816: $57-66$.

22. D'Aguanno S, Barcaroli D, Rossi C, Zucchelli M, Ciavardelli D, Cortese C et al. P63 isoforms regulate metabolism of cancer stem cells. J Proteome Res 2014; 13: 2120-2136.

23. Konecny GE, Pegram MD, Venkatesan N, Finn R, Yang G, Rahmeh M et al. Activity of the dual kinase inhibitor lapatinib (GW572016) against HER-2-overexpressing and trastuzumabtreated breast cancer cells. Cancer Res 2006; 66: 1630-1639.

24. Lowery AJ, Miller N, McNeill RE, Kerin MJ. MicroRNAs as prognostic indicators and therapeutic targets: potential effect on breast cancer management. Clin cancer Res 2008; 14: 360-365.

25. Wiemer EAC. The role of microRNAs in cancer: no small matter. Eur J Cancer 2007; 43: 1529-1544

26. Avril-Sassen S, Goldstein LD, Stingl J, Blenkiron C, Le Quesne J, Spiteri I et al. Characterisation of microRNA expression in post-natal mouse mammary gland development. BMC Genomics 2009; 10: 548 .

27. Sempere LF, Christensen M, Silahtaroglu A, Bak M, Heath C V, Schwartz G et al. Altered MicroRNA expression confined to specific epithelial cell subpopulations in breast cancer. Cancer Res 2007; 67: 11612-11620.

28. Ibarra I, Erlich Y, Muthuswamy SK, Sachidanandam R, Hannon GJ. A role for microRNAs in maintenance of mouse mammary epithelial progenitor cells. Genes Dev 2007; 21: 3238-3243.

29. Greene S, Herschkowitz J, Rosen J. The ups and downs of miR-205: identifying th eroles of miR-205 in mammary gland development and breast cancer. RNA Biol 2011; 7: 300-304.

30. Danilov AV, Neupane D, Nagaraja AS, Feofanova EV, Humphries LA, DiRenzo J et al. DeltaNp63alpha-mediated induction of epidermal growth factor receptor promotes pancreatic cancer cell growth and chemoresistance. PLoS One 2011; 6: e26815.

31. Yu J, Ryan DG, Getsios S, Oliveira-Fernandes M, Fatima A, Lavker RM. MicroRNA-184 antagonizes microRNA-205 to maintain SHIP2 levels in epithelia. Proc Natl Acad Sci USA 2008; 105: 19300-19305

32. Iorio MV, Casalini P, Piovan C, Di Leva G, Merlo A, Triulzi T et al. microRNA-205 regulates HER3 in human breast cancer. Cancer Res 2009; 69: 2195-2200.
33. Wang D, Zhang Z, O'Loughlin E, Wang L, Fan X, Lai EC et al. MicroRNA-205 controls neonatal expansion of skin stem cells by modulating the PI3K pathway. Nat Cell Biol 2014; 15: $1153-1163$.

34. Todaro M, Alea MP, Di Stefano AB, Cammareri P, Vermeulen L, lovino F et al. Colon cancer stem cells dictate tumor growth and resist cell death by production of interleukin-4. Cell Stem Cell 2007; 1: 389-402.

35. Lena AM, Shalom-Feuerstein R, Rivetti di Val Cervo P, Aberdam D, Knight RA, Melino G et al. miR-203 represses "stemness" by repressing DeltaNp63. Cell Death Differ 2008; 15: 1187-1195.

36. Candi E, Terrinoni A, Rufini A, Chikh A, Lena AM, Suzuki $Y$ et al. p63 is upstream of IKK alpha in epidermal development. J Cell Sci 2006; 119: 4617-4622.

37. Ricci-Vitiani L, Pedini F, Mollinari C, Condorelli G, Bonci D, Bez A et al. Absence of caspase 8 and high expression of PED protect primitive neural cells from cell death. J Exp Med 2004; 200: 1257-1266.

38. Rocco JW, Leong CO, Kuperwasser N, DeYoung MP, Ellisen LW. p63 mediates survival in squamous cell carcinoma by suppression of p73-dependent apoptosis. Cancer Cell 2006; 9 : 45-56.

39. Follenzi A, Ailles LE, Bakovic S, Geuna M, Naldini L. Gene transfer by lentiviral vectors is limited by nuclear translocation and rescued by HIV-1 pol sequences. Nat Genet 2000; 25 : 217-222.

40. De Cola A, Bongiorno-Borbone L, Bianchi E, Barcaroli D, Carletti E, Knight RA et al. FLASH is essential during early embryogenesis and cooperates with $\mathrm{p} 73$ to regulate histone gene transcription. Oncogene 2012; 31: 573-582.

41. Cheang MC, Chia SK, Voduc D, Gao D, Leung S, Snider J et al. Ki67 index, HER2 status, and prognosis of patients with luminal B breast cancer. J Natl Cancer Inst 2009; 101: $736-750$.

(i) Cell Death and Disease is an open-access journal published by Nature Publishing Group. This work is licensed under a Creative Commons Attribution 4.0 International License. The images or other third party material in this article are included in the article's Creative Commons license, unless indicated otherwise in the credit line; if the material is not included under the Creative Commons license, users will need to obtain permission from the license holder to reproduce the material. To view a copy of this license, visit http://creativecommons.org/licenses/by/4.0/

Supplementary Information accompanies this paper on Cell Death and Disease website (http://www.nature.com/cddis) 